\section{Avanzando hacia la vida independiente: planteamientos educativos en jóvenes con discapacidad intelectual}

\section{Moving towards independent life: Educational approaches in young people with intellectual disability}

\section{Resumen}

El avance de los jóvenes con discapacidad intelectual hacia mayores desarrollos de su autonomía personal requiere de nuevos planteamientos educativos, centrados en la autodeterminación, la inclusión social y la vida independiente. Down España, en colaboración con Down Huesca y Down Lleida, ha desarrollado un proyecto que va en esta dirección. El proyecto formativo se ha contextualizado dentro de los cambios que se producen en la concepción de la discapacidad. Autodeterminación, calidad de vida, modelos de apoyos, modelo social y accesibilidad universal son algunos de los conceptos que guían nuestro proyecto y que requieren nuevos enfoques organizacionales y nuevas prácticas profesionales y parentales. Los módulos y talleres tienen como referente los proyectos de vida independiente. La metodología didáctica que se desarrolla en la aplicación de los programas está fundamentada en el modelo didáctico mediacional, en el aprendizaje cooperativo, en el enfoque globalizado y en la generalización de los aprendizajes.

\section{Palabras clave}

Síndrome de Down, discapacidad intelectual, autodeterminación, inclusión social, vida independiente.

\section{Abstract \\ The advance of young people with intellectual disability towards greater development of their personal autonomy requires new educational approaches, based on the self- determination, social inclusion and independent life. Down España, in collaboration with Down Huesca and Down Lleida, has developed \\ Elías Vived Conte <evived@unizar.es> \\ Asociación Down Huesca \\ Eva Betbesé Mullet \\ Asociación Down Lleida \\ Mónica Díaz Orgaz \\ Down España} a project based on this premise. The formative project has been contextualized within the changes that occur in the conception of disability. Self-determination, quality of life, support models, social model, universal accessibility, etc., are some of the concepts that guide our project and require new organizational approaches and new professional and parental practices. The modules and workshops have the independent life projects as referent. The teaching methodology developed in the implementation of the programs is based on the mediation teaching model, as well as on the cooperative learning, the globalized approach and on the generalization of the learning.

\section{Keywords:}

Down syndrome, intellectual disability, self-determination, social inclusion, independent life.

\section{Adriana González-Simancas Sanz}

Down España

\section{Agustín Matía Amor}

Down España

\author{
Para citar: \\ Vived Conte, E. et al. (20I3): \\ "Avanzando hacia la vida \\ independiente: planteamientos \\ educativos en jóvenes con discapacidad \\ intelectual", Revista Española de \\ Discapacidad, \\ I (I): II9-I38. \\ doi: <http://dx.doi.org/IO.5569/2340- \\ 5104.01.01.06>
}

Fecha de recepción: I2-I I-2OI 2 Fecha de aceptación: 22-4-20I3 


\section{Introducción}

La promoción de la autonomía y la independencia sigue siendo uno de los retos más importantes para las personas con síndrome de Down o con otra discapacidad intelectual. Resulta fundamental capacitar a estas personas para que puedan llevar una vida más autónoma e independiente, y ello debe plantearse a lo largo de toda la vida. Cuando hablamos de vida independiente, nos estamos refiriendo a que la persona sea dueña de su propia vida, que decida sobre las cosas que le preocupan y le interesan.

En este sentido, las personas con síndrome de Down y sus familias se expresaron recientemente, en el XII Encuentro Nacional de Familias y IV Encuentro Nacional de Hermanos de Personas con Síndrome de Down, indicando sus retos actuales. En efecto, más de 500 personas acudieron a estos encuentros, organizados por Down España y Down Huesca, que se celebraron en Huesca entre el I y 4 de noviembre de 20 I 2. Se organizaron varias mesas redondas cuyas temáticas iban en esta dirección: los servicios de promoción de autonomía personal; la autodeterminación, la planificación centrada en la persona y el papel de la familia; la formación para la inclusión social y vida independiente; o el papel de las familias como mediadoras de la vida autónoma, inclusiva e independiente de sus hijos con síndrome de Down.

Jóvenes con síndrome de Down participaron en diversos talleres durante el encuentro, donde ejercieron como protagonistas de su vida. Expresaron que, si quieren alcanzar mayores cotas de autonomía, necesitan tomar sus propias decisiones en este y otros aspectos vitales. Estos jóvenes con síndrome de Down dejaron claro que se preparan con empeño para autorrealizarse como personas autónomas, y que disfrutan comprobando cómo crecen sus responsabilidades y posibilidades. Destacaron que durante mucho tiempo han tenido que confiar en la familia y asociaciones para que se expresaran por ellos, pero que hoy día poseen la suficiente independencia y autonomía para expresarse en libertad y reclamar sus derechos innegables. Tan sólo pidieron que la sociedad reflexione un poco y se pare a escucharles, para que comprenda que son ciudadanos de pleno derecho. Precisamente, una de las conclusiones del encuentro indicaba que, gracias al esfuerzo y reivindicaciones de las personas con discapacidad, estamos dirigiéndonos a una nueva concepción de la persona con síndrome de Down, donde destaca su condición humana, su dignidad, su participación igualitaria en la vida de la comunidad, y donde cuenta con una red de apoyos personalizados. Es un sujeto con capacidad y derechos para ejercer el control de su vida en todos los ámbitos.

Capacitar para la vida autónoma e independiente supone plantear una orientación educativa que, iniciada en la familia, se extienda a lo largo de los años en los diferentes escenarios de aprendizaje por los que discurre la persona (centros escolares, asociaciones, centros de formación laboral, otros centros educativos). Dicha orientación educativa debe incidir en el desarrollo de la autonomía y la vida independiente, en la inclusión social y en la autodeterminación.

En esta dirección, se ha comenzado a desarrollar una estrategia, en el seno de Down España, orientada a indagar fórmulas educativas que se centren en los aspectos mencionados. Así, además de poner en marcha un conjunto de redes nacionales (entre las que se encuentran la Red Nacional de Educación y la Red Nacional de Vida Independiente) que ofrecen oportunidades para un trabajo colaborativo de las entidades asociadas, durante 20 I I se inició un camino de revisión de la estructura curricular dirigida a los jóvenes con síndrome de Down o con otras discapacidades intelectuales. Dicho cambio se producía a partir de diversas experiencias que estaban realizando adultos con discapacidad intelectual en diferentes localidades, especialmente vinculadas a la inclusión laboral en empresas y a la vivienda independiente (con los apoyos adecuados a dichas situaciones).

Vived et al. (20 г 2a), en un artículo descriptivo del Programa de Formación para la Inclusión 
Social y la Vida Independiente, consideran que dicho proyecto:

\section{Constituye una experiencia pionera, que surge de las necesidades del colectivo de personas con discapacidad intelectual, analizadas a la luz de las nuevas formas de conceptualizar la discapacidad, de considerar las opiniones de los propios jóvenes con discapacidad, de sus padres y de los profesionales de las asociaciones.}

En dicho artículo, se explica que este proyecto fue presentado a la convocatoria del Ministerio de Educación para la realización de actuaciones dirigidas a la atención del alumnado con necesidad específica de apoyo educativo y a la compensación de desigualdades en educación, y fue aprobado para ser desarrollado durante el curso 2OI I-I 2. Estuvo coordinado por Down España y se llevó a cabo, en una primera fase piloto, en Down Huesca y Down Lleida. Actualmente, se está desarrollando una nueva fase, que se desarrollará durante el curso 20I2-I3, a la que también se ha incorporado Down Burgos.

Las actuaciones definidas en dicho programa van encaminadas a contribuir a que las personas con discapacidad intelectual consigan acceder al mercado de trabajo, participen activamente en la comunidad, en un avance decidido hacia la inclusión social, y disfruten de la opción, si así lo desean, de vivir de forma independiente, con los apoyos (profesionales o naturales) que en cada caso sean precisos. Lógicamente, esta propuesta formativa, que se desarrolla en las asociaciones, es complementaria a la atención educativa que los jóvenes reciben en los centros escolares.

Además de las actuaciones dirigidas a las personas con discapacidad, también se desarrollaron en el proyecto otras actuaciones dirigidas a las familias, a los profesionales de las asociaciones y de los centros escolares, o a los voluntarios. Resulta fundamental diseñar nuevos proyectos que faciliten el acceso al empleo, la participación social y la vida autónoma, y que contribuyan a que los jóvenes con discapacidad intelectual puedan vivir de una manera más independiente y disfruten de la mayor calidad de vida posible en entornos inclusivos.

El proyecto formativo que hemos desarrollado se contextualiza dentro de los cambios que se producen en las normativas (particularmente la Convención Internacional de los Derechos de las Personas con Discapacidad) y en la concepción de la discapacidad en general y de la discapacidad intelectual en particular. Autodeterminación, calidad de vida, modelos de apoyos, modelo social de la discapacidad, accesibilidad universal y diseño para todos, inclusión social y vida independiente son algunos de los conceptos que han guiado nuestro proyecto. Asimismo, lo hemos vinculado con los itinerarios personalizados hacia la vida independiente.

En este contexto de cambio, se exigen nuevos marcos de comprensión, nuevas actitudes hacia la discapacidad desde el respeto a las diferencias y la igualdad de oportunidades de todos los hombres y mujeres, nuevos enfoques organizacionales, nuevas prácticas profesionales y parentales (Vived et al. 20 I 2b: 2). El proyecto que hemos elaborado reflexiona sobre todas estas cuestiones.

En definitiva, el proyecto se inscribe dentro de las medidas que garantizan la igualdad de oportunidades y la accesibilidad a entornos inclusivos (en el trabajo, en la vivienda, en la comunidad, en la formación). Y se plantea como una formación complementaria a la realizada en los centros docentes sostenidos con fondos públicos. Esta formación se lleva a cabo en las asociaciones y en contextos comunitarios, y el proceso desarrollado se analiza a través de la metodología de investigación-acción. Se han mantenido reuniones periódicas con los centros educativos, de modo que el profesorado tiene información puntual del desarrollo del proyecto, con la finalidad de ir incorporado en los diseños curriculares de las últimas etapas educativas nuevos objetivos y contenidos vinculados con los nuevos conocimientos sobre discapacidad intelectual (que se deriven de esta y de otras investigaciones y prácticas), con nuevos proyectos vitales, nuevas expectativas y nuevos planteamientos pedagógicos. 
Los módulos y talleres que se han diseñado tienen como referente los proyectos de vida independiente (vivienda con apoyo, empleo con apoyo, participación ciudadana, formación permanente). La metodología didáctica que se ha desarrollado en la aplicación de los programas está fundamentada en el modelo didáctico mediacional, en el aprendizaje cooperativo, en el enfoque globalizado y en la generalización de los aprendizajes.

Este proyecto tiene un marcado carácter innovador, y ha tratado de poner en práctica y 'traducir' en actividades cotidianas la nueva conceptualización sobre discapacidad, las nuevas normativas y los nuevos planteamientos sobre los proyectos de vida de las personas con síndrome de Down o con otras discapacidades intelectuales.

\section{Marcos referenciales del proyecto}

El plan de formación que se propone en el proyecto se fundamenta en los nuevos conceptos sobre discapacidad; también pretende orientarse a partir de los derechos fundamentales de las personas con discapacidad. Por otro lado, hemos considerado como referentes curriculares del programa educativo tanto los proyectos de vida independiente como la necesaria cooperación de la familia, destacando su papel primordial en el desarrollo de la autonomía de los jóvenes. Sobre estos aspectos, la nueva conceptualización de la discapacidad en general y de la discapacidad intelectual en particular (contexto teórico) y los referentes curriculares del proyecto (contexto operativo), van a tratar los epígrafes siguientes.

\subsection{Fundamentación teórica: nuevos enfoques sobre la discapacidad}

En nuestro anterior trabajo (Vived et al., 20I 2a), ya expusimos que el proyecto educativo pretendía contextualizarse, en la dimensión conceptual, dentro de los nuevos enfoques sobre la discapacidad: modelo de apoyos, autodeterminación, calidad de vida, accesibilidad universal, diseño para todos o vida independiente. Estos conceptos constituyen un nuevo modo de entender la discapacidad y, lo que es más importante, de ellos se derivan implicaciones importantes en los ámbitos educativos, laborales y sociales, así como cambios en las organizaciones y en los servicios de atención a las personas con discapacidad. La conceptualización de la discapacidad va a influir en la percepción que tenemos sobre el papel que las personas con discapacidad deben jugar en el trabajo y en la sociedad, y tiene implicaciones en el modo de actuar y relacionarnos con ellas, en las prácticas parentales y profesionales, $\mathrm{y}$ en la promoción de determinados programas educativos, que deben estar más centrados en la autonomía y la vida independiente, en la autodeterminación y en la inclusión social.

La discapacidad no puede identificarse exclusivamente como una característica del individuo, sino que debe entenderse como un estado del funcionamiento de la persona, que depende no sólo de las condiciones individuales, sino que está muy influido por las oportunidades que tiene la persona para desarrollarse, así como por los apoyos que se le ofrecen para facilitar tal desarrollo. Ahora bien, ambos aspectos, oportunidades y apoyos, dependen del contexto, de la conceptualización que se tiene de la discapacidad intelectual y del modelo de intervención que se plantea con ella.

Los nuevos enfoques sobre discapacidad, que van a ser determinantes en el desarrollo del proyecto educativo, podemos concretarlos en los siguientes:

- Evolución del concepto de discapacidad intelectual: modelo de apoyos de la Asociación Americana de Discapacidades Intelectuales y del Desarrollo (Luckasson et al., 2002; Schalock et al., 2010; Verdugo, 20IO).

- Calidad de vida (Schalock, I996; Van Loon, 2008).

- Autodeterminación y autonomía personal (Wehmeyer, I996; Wehmeyer et al., 2006). 
- Accesibilidad universal, adaptabilidad de los contextos y diseño para todos (Alonso, 2007; Ginnerup, 2010).

- Vida independiente (Vidal, 2003; Foro de Vida Independiente).

- Modelo social de la discapacidad (Jiménez, 2007; Verdugo, I995).

Uno de los principales cambios derivados de estas perspectivas es el énfasis creciente en el reconocimiento de las personas con discapacidad como agentes y beneficiarios del desarrollo de las sociedades en que viven, en lugar de considerarlas únicamente desde la óptica de los servicios biomédicos y el bienestar social. La preocupación ha pasado a centrarse en los factores (sociales, económicos, institucionales y formativos) que determinan el entorno en que se desenvuelven las personas con discapacidad, y en la importancia de eliminar obstáculos y promover entornos accesibles para que las personas con discapacidad puedan mejorar su participación en la vida social y económica.

\subsection{Referentes curriculares: los proyectos de vida independiente y la colaboración con las familias}

\subsubsection{Los proyectos de vida independiente}

Si los ámbitos conceptuales, analizados en el epígrafe anterior, influyen en el planteamiento del proyecto formativo que presentamos, los proyectos de vida independiente suponen el principal referente que consideramos para determinar el programa de formación para personas con discapacidad. En los últimos años, se está avanzando de manera intensa hacia nuevos modelos de intervención cuya finalidad es el desarrollo de los proyectos de vida independiente.

El objetivo básico del proyecto de vida independiente es ofrecer oportunidades y apoyos que fomenten el desarrollo de habilidades sociales, de autonomía personal, de autorregulación y de autodeterminación de las personas con capacidades diversas, además de mejorar su autoestima y su calidad de vida, acercándolas cada vez más a la normalización plena en todos los ámbitos personales y en diferentes contextos (laboral, de la vivienda, social, formativo).

El proyecto de vida independiente para personas con discapacidad intelectual, promovido a partir del modelo social, de la autodeterminación y de la inclusión social, se puede concretar en los siguientes componentes: participación laboral (acceso al empleo), vivienda compartida (emancipación de la familia), participación ciudadana (inclusión social) y formación permanente (en entornos inclusivos). Por otro lado, la autodeterminación es una de las ideas fundamentales que debe regir el funcionamiento de todos los ámbitos del proyecto y constituye un concepto clave a la hora de entender la expresión libre de cualquier persona.

El proceso educativo que van a seguir los participantes en el plan formativo que se impulsa se ha planteado como un medio para que aprendan a desenvolverse con autonomía y responsabilidad en la comunidad, en el hogar y en el trabajo. Que conozcan y se impliquen en los diferentes ámbitos y oportunidades que ofrece la comunidad (en lo cultural, en lo deportivo, en lo institucional, en lo lúdico); que compartan momentos agradables en actividades recreativas o de ocio, con personas con y sin discapacidad; que se manejen con destreza en las diferentes tareas del hogar; que desarrollen habilidades de comunicación y convivencia con otras personas; que organicen su tiempo dentro de su vivienda; que desempeñen trabajos en el mundo laboral integrado. Éstas son algunas de las condiciones necesarias para desarrollar una adecuada inclusión social de las personas con discapacidad.

El proyecto de vida independiente pretende avanzar en estos desarrollos y favorecer la generalización de la autonomía personal de las personas con discapacidad en todos los ámbitos, preparándolas para llevar una vida más autónoma e independiente.

Además del impulso en el desarrollo de determinados ámbitos contextuales (vivienda, 
trabajo, comunidad, formación), que van a permitir el desarrollo de itinerarios individuales de vida independiente, basados en la autodeterminación y en la inclusión social, se requiere también una formación específica para la inclusión social y la vida independiente, que se presente con los nuevos planteamientos teóricos que hemos definido y con una metodología didáctica mediacional, basada en el sentimiento de competencia, en los procesos y en la generalización de los aprendizajes. Dicha formación debe ir dirigida tanto a las personas con síndrome de Down o con otra discapacidad intelectual, como a sus familias y a los profesores y técnicos que se relacionan con ellas.

\subsubsection{El papel de las familias. Marcos de cooperación}

La estrecha colaboración de los padres constituye una seña de identidad del proyecto de formación. La participación de los padres constituye un elemento esencial para el éxito de la intervención educativa en los niños y jóvenes con discapacidad intelectual. Los padres son los primeros y principales agentes de la educación de su hijo, y juegan un rol primordial desde su edad temprana: son las personas que disponen de más oportunidades para influir en el comportamiento de sus hijos y favorecer así su desarrollo. Que los padres se integren en el proceso de la educación de sus hijos permitirá optimizar la intervención educativa. Son enormes las ventajas de la participación activa de los padres en los programas educativos de los jóvenes con discapacidad intelectual. Cuando los padres están implicados en los programas de intervención, el mantenimiento y generalización de los aprendizajes hechos por el niño o el joven tienen más posibilidades de producirse y consolidarse.

En el presente programa se plantea la colaboración de los padres en tres ámbitos importantes: en el diseño de los programas educativos (colaborando en la definición de los proyectos de vida independiente $y$ en las repercusiones que tienen sobre las prácticas parentales), en la aplicación de los programas (colaborando en la consolidación y generalización de los aprendizajes), y en la valoración del desarrollo del hijo/a y del propio programa (no sólo serán útiles sus observaciones sobre la evolución de su hijo/a, sino también sus apreciaciones sobre el desarrollo del programa en los diferentes contenidos).

\section{Objetivos y actividades}

Los objetivos que se han planteado en el proyecto han sido los siguientes:

- Proponer un proyecto educativo para jóvenes con síndrome de Down o con otras discapacidades intelectuales que se centre en el desarrollo de los proyectos de vida independiente.

- Aplicar y evaluar el programa formativo en una muestra de alumnos/as con discapacidad intelectual.

- Indagar nuevos planteamientos didácticos, coherentes con los perfiles de aprendizaje de los alumnos.

- Diseñar y aplicar acciones formativas para las familias, así como acciones colaborativas entre padres y profesionales, que tengan como referencia la nueva conceptualización de la discapacidad intelectual.

- Establecer mecánicas sensibilizadoras y formativas de todos los agentes educativos que intervienen con este alumnado (directivos y técnicos de las asociaciones, profesorado de centros educativos, voluntarios y estudiantes en prácticas que colaboran en el desarrollo del proyecto).

- Desarrollar mecanismos de colaboración entre las asociaciones de síndrome de Down y los centros educativos para ofrecer una respuesta educativa integral y coordinada, centrada en la satisfacción de las necesidades educativas de los alumnos con discapacidad intelectual, y coherente con los nuevos conocimientos y conceptos sobre discapacidad en general y discapacidad intelectual en particular. 
- Divulgar el proceso seguido y los resultados obtenidos en el proyecto a las asociaciones de la Red de Educación Inclusiva de Down España, así como a los centros educativos que tienen integrados a los alumnos con discapacidad intelectual.

En el proyecto se plantearon distintos tipos de actuaciones, relacionadas con los objetivos señalados. Fueron las siguientes:

- Acciones de diseño, elaboración y planificación.

- Actuaciones realizadas con los alumnos.

- Actuaciones desarrolladas con la familia.

- Actuaciones con profesionales de las asociaciones, y con los voluntarios y alumnado en prácticas que han colaborado en el proyecto.

- Actuaciones con los centros escolares.

- Actuaciones de investigación e innovación.

- Actuaciones de elaboración de material didáctico.

- Actuaciones de divulgación y multiplicación de efectos.

\section{Planteamientos didácticos}

Las orientaciones didácticas que van a guiar la aplicación de los distintos programas o talleres están basadas en el trabajo de Molina et al. (2008a), que presenta un modelo didáctico validado en una muestra de niños con síndrome de Down y que ya se ha utilizado en la aplicación de distintos programas -un programa de habilidades sociales, autonomía personal y autorregulación (Vived, 20I I), y un programa de autodeterminación, participación social y participación laboral (Vived et al. 20 2 2b)-. A continuación, se presentan los principios didácticos que se derivan del modelo señalado y que han servido tanto para el diseño de las unidades didácticas de los diferentes módulos y programas como para su desarrollo; también se analiza la mediación en la interacción profesoralumno. Por el interés que le hemos dado al 'cómo enseñar', nos ha parecido oportuno exponer en detalle algunos de los planteamientos didácticos.

\subsection{Principios didácticos}

Los principios didácticos que han servido tanto para el diseño de las unidades didácticas como para su desarrollo se pueden concretar en los siguientes: principio de globalización, programación basada en la zona de desarrollo próximo, mediación como estrategia de enseñanza (basada en la experiencia de aprendizaje mediado), aprendizaje cooperativo y generalización de los aprendizajes.

\subsubsection{El principio de globalización: eje vertebrador de la metodología didáctica}

En los diferentes programas educativos, se pretende agrupar los contenidos según el principio de la concentración, consistente en la ordenación de los ámbitos relacionados con las actividades que se van a trabajar en torno a intereses específicos y operantes en la vida del alumno. A partir de este principio, se integran diversos contenidos en una misma unidad didáctica. Por medio de un centro de interés, las clases se realizan abarcando un grupo de áreas que se van desarrollando a través de las diferentes actividades que se proponen. Por otro lado, también se organizan los contenidos de aprendizaje para que sirvan a la solución de problemas reales, que respondan a los intereses de los alumnos, a sus experiencias, vivencias y necesidades.

\subsubsection{La zona de desarrollo próximo, base de la programación didáctica}

Vigotsky (1964, I979) definió la zona de desarrollo próximo como el espacio que hay entre lo que la persona es capaz de realizar por sí misma (nivel de desarrollo efectivo o actual) y lo que es capaz de llevar a cabo con ayuda de algún mediador (nivel de desarrollo potencial). Actualmente constituye un constructo que tiene una gran utilidad en el ámbito psicopedagógico. 
Una programación didáctica fundamentada en la zona de desarrollo próximo requiere, por ejemplo, que el nivel de dificultad de las actividades propuestas se corresponda con el nivel de desarrollo potencial del alumno y no con el nivel de desarrollo efectivo o actual, lo cual exige, a su vez, que los objetivos y los contenidos tengan también como referencia dicho nivel de desarrollo potencial.

El profesorado que intente realizar sus programaciones didácticas de acuerdo con las exigencias de la zona de desarrollo próximo tiene que ser muy consciente de que, antes de exigir a sus alumnos que resuelvan las tareas de forma independiente, es necesaria una intervención metodológica que respete los parámetros de la experiencia de aprendizaje mediado.

\subsubsection{La mediación como estrategia de enseñanza}

La metodología mediacional se centra en los procesos más que en los productos o resultados, pretende favorecer la transferencia de los principios cognitivos a distintos ámbitos de aplicación (familiar, escolar, grupo de amigos), incide en comunicar entusiasmo por el aprendizaje, en relacionar las experiencias nuevas con las familiares y en desarrollar el sentimiento de competencia de los alumnos.

Diversas teorías del aprendizaje y del desarrollo cognitivo han abordado el tema de la mediación. Autores como Vigotsky, Feuerstein y Haywood, entre otros, han analizado la mediación como estrategia de enseñanza. Haywood et al. (I992) consideran que la mayoría de las interacciones entre niños y adultos son potencialmente experiencias de aprendizaje mediadas.

Feuerstein (I996) define la experiencia del aprendizaje mediado como una cualidad de la interacción ser humano-entorno, que resulta de los cambios introducidos en esta interacción por un mediador humano que se interpone entre el organismo receptor y las fuentes de estímulo. El mediador selecciona, organiza y planifica los estímulos, variando su amplitud, frecuencia e intensidad, y los transforma en poderosos determinantes del comportamiento. El mediador proporciona al individuo un aprendizaje organizado y estructurado

\subsubsection{El aprendizaje cooperativo, base de la experiencia de aprendizaje mediado}

Molina et al. (2008b) señalan que la situación de aprendizaje cooperativo es connatural a la experiencia de aprendizaje mediado y a la programación didáctica basada en la zona de desarrollo próximo, pero no es la única situación de aprendizaje que conviene poner en práctica. El modelo didáctico, que los autores citados consideraban más útil para los alumnos, requiere estas tres situaciones de aprendizaje: gran grupo (en esta situación, el papel preponderante del proceso didáctico corresponde al profesor), pequeño grupo (es la situación propia del aprendizaje cooperativo) y trabajo individual (es el que permite la consolidación de la organización cognitiva). Es decir, el aprendizaje cooperativo es una situación de aprendizaje posible y recomendable, pero no debe ser la única.

\subsubsection{Generalización de los aprendizajes: papel de los padres en una red coordinada}

Los bajos niveles de consolidación y generalización de los aprendizajes se encuentran entre las dificultades más notables de las personas con discapacidad intelectual. Para abordar esta cuestión, en el proyecto se planteó un sistema de intervención basado en la configuración de una red coordinada de agentes educativos (profesorado, padres y profesionales de otras entidades) que, desde una perspectiva de interdependencia solidaria, permita una mayor eficacia de las actuaciones educativas. El papel de los padres resulta fundamental para facilitar la generalización de los aprendizajes. Por otro lado, una técnica eficaz en el desarrollo de generalización es la definida por Haywood como una aplicación o un puenteo a través del cual se pretende vincular los aprendizajes desarrollados en clase con otros ámbitos o 
contextos. Al desarrollo de estos dos aspectos se dirigen las líneas siguientes.

\subsubsection{La aplicación como técnica de mediación y de generalización}

Haywood et al. (I992) consideran la aplicación como una importante herramienta en la enseñanza mediacional. Como técnica de la enseñanza mediacional, la aplicación (bridging) implica hablar sobre cómo y cuándo utilizar nuevos conceptos, relaciones, técnicas o destrezas en una amplia gama de contextos. Se trata, por tanto, de conectar principios y estrategias con aplicaciones. Cada nueva aplicación contribuye a definir los tipos de situaciones en los que un principio determinado puede o no aplicarse, y ayudarnos así a distinguir un principio de otro tal vez similar. Además, la práctica en la aplicación de modos de pensar recién aprendidos fomenta la utilización espontánea que el alumno hace de tales aplicaciones.

Los profesores mediacionales pueden enseñar importantes modos de pensar y estrategias de resolución de problemas por medios orales, mediante demostración, planteando problemas y solicitando soluciones (y explicaciones sobre esas soluciones). Además discuten con los alumnos una variedad de aplicaciones en distintos ámbitos de sus vidas; es decir, 'extraen' de ellos aplicaciones que sirvan a modo de 'puentes' entre los mismos procesos de pensamiento (en abstracto) y sus aplicaciones concretas a diversas situaciones (otras actividades escolares, vida hogareña y familiar, actividades recreativas o entre el grupo de amigos).

\subsubsection{Papel de los padres en los programas educativos}

El alumno no aprende sólo en la escuela: las experiencias en el hogar, con sus padres y hermanos y aquellas que tiene con sus amigos van a ejercer una enorme influencia sobre su aprendizaje. El alumno va a beneficiarse de numerosas actividades de desarrollo que se llevan a cabo en casa. Así, el desarrollo de su autonomía personal, de su capacidad comunicativa, de su socialización, entre otras cuestiones, van a estar influenciadas, de un modo decisivo, por la labor de la familia.

En el desarrollo de la autodeterminación, de la autonomía personal y de las competencias para una vida independiente, la familia va a jugar un papel fundamental, porque, a pesar de que las influencias externas son cada vez más numerosas, la familia constituye la institución básica en el desarrollo y la educación de los alumnos. El desarrollo de la autodeterminación, de la autonomía personal y de la competencia social requiere de un sistema de apoyo que favorezca tal desarrollo, que favorezca las iniciativas de la persona y su participación en las acciones relevantes para su vida, que promueva el establecimiento de metas personales, que ayude a la persona a estar segura de sí misma, a confiar y a valorar sus logros, que potencie la autonomía. Ese sistema debe construirse de modo colaborativo entre padres y profesionales. En este sentido, es necesario articular actividades que traten de coordinar los diferentes escenarios, específicamente el escolar, el familiar y el asociativo.

\subsection{El profesor como mediador}

Por el interés que tiene la mediación como estrategia de enseñanza, nos parece oportuno establecer algunas consideraciones sobre el papel del profesor como mediador. Un aspecto importante de la mediación lo constituye el estilo docente mediacional, que es el modo que tiene el profesor de interactuar con los alumnos para desarrollar procesos de aprendizaje. $\mathrm{La}$ mediación capacita a los alumnos para aprender procesos, más que contenidos de aprendizaje. Para facilitar el aprendizaje, el profesor ha de manifestar las siguientes conductas, consideradas como líneas directrices de su actuación (Haywood et al. I992):

- Destacar las interacciones entre los alumnos durante las situaciones de juego.

- Explicitar la evidencia del pensamiento de los alumnos. 
- Plantear cuestiones y preguntas sobre los procesos seguidos por los alumnos, destacando sobre todo el proceso utilizado, y no el producto o respuesta.

- Animar al alumno a razonar todas sus respuestas. Exigirle una justificación, incluso para las respuestas correctas.

- Potenciar en el alumno la formulación de reglas y conclusiones de los ejemplos estudiados.

- Crear confianza en el niño, fomentando el sentimiento de su competencia cognitiva.

- Relacionar los procesos de pensamiento de cada unidad didáctica con la aplicación de aquéllos. Transferir los principios a los distintos ámbitos de contenido familiar o escolar.

- Relacionar las experiencias nuevas con las ya conocidas.

- Favorecer el establecimiento de razonamientos lógicos y aceptar tantas respuestas de los alumnos como sea posible, corrigiendo las incorrectas o imprecisas.

Con estas acciones, el profesor ayuda al alumno a organizar sus observaciones sobre los procesos de pensamiento que ha puesto en funcionamiento para resolver la tarea y a analizar la aplicabilidad de éstos a otras situaciones. Para que una interacción entre un profesor (u otro mediador) y un alumno pueda considerarse como interacción mediada y potenciadora del desarrollo cognitivo, debe guiarse por algunos de los criterios que se relacionan a continuación, ya señalados por Feuerstein (I986) y citados, algunos de ellos, por Haywood et al. (I992) y por Prieto (I989):

- Intencionalidad y reciprocidad.

- Transcendencia.

- Comunicación del significado y propósito cognitivo.

- Mediación de los sentimientos de competencia.

- Regulación y control de la conducta.

- Participación activa y conducta compartida.

- Individualización y diferenciación psicológica.

- Mediación de la búsqueda, planificación y logro de objetivos de conducta.
- Adaptación a la novedad.

- Mediación del conocimiento de la modificabilidad y del cambio.

- Transmisión mediada de valores y actitudes.

5. Evaluación y seguimiento de las acciones

Uno de los aspectos más importantes de un programa educativo es el diseño del procedimiento de evaluación. La actividad educativa es enormemente compleja y exige una constante evaluación de su desarrollo y de sus resultados, con mayor énfasis si el programa está sujeto a un proceso de indagación experimental. Pero la evaluación no debe considerarse únicamente como un elemento final del proceso didáctico, sino que debe constituirse como un componente sustancial de aquél, desde el comienzo y durante su desarrollo, y realizarse, por tanto, de forma continuada y no de modo circunstancial. Hay que tener presente que la evaluación debe proporcionar una información continuada, objetiva y suficiente, que permita la regulación de los procesos educativos de forma eficiente y continua.

Por otro lado, la evaluación debe extenderse a todo el programa educativo y no sólo a los alumnos. Tiene que cumplir funciones de regulación activa de los diferentes elementos del programa y de los procesos educativos, y no solamente centrarse en la valoración de los alumnos. En el proyecto se propone un sistema de valoración del programa centrado en los estándares de calidad.

Con respecto a la evaluación del funcionamiento de los alumnos, utilizamos diversos instrumentos de evaluación continua: registros del desarrollo de las sesiones, cuestionarios para la valoración continua del funcionamiento de los alumnos y cuestionarios de autoevaluación.

Con respecto a la valoración del programa, se plantea un sistema de evaluación basado en los estándares profesionales. Actualmente, hay una 
preocupación creciente por la calidad. El diseño y la aplicación de los programas educativos no deben ser ajenos a esta tendencia orientada a la calidad, cuya meta es incrementar la eficacia, la competencia y la satisfacción de los alumnos. La calidad de los servicios y programas que una organización pone en marcha para atender a las necesidades específicas de determinados colectivos es uno de los retos más importantes que puede afrontarse en la actualidad. Es necesario avanzar en una cultura profesional centrada en la calidad y que se base en las coincidencias prácticas que muestran grupos de profesionales.

En este contexto, se hace necesario determinar una relación de estándares profesionales que concreten y garanticen unos parámetros básicos de intervención, ponga en práctica los conocimientos adquiridos, y sirva de elemento de contraste y evaluación de resultados para todas las partes implicadas en los procesos educativos. Podemos definir los estándares como criterios de calidad que permiten contrastar la práctica profesional. Estos principios o criterios de calidad deben ser acordados por un grupo significativo de profesionales.

\section{Población destinataria}

Ya se ha comentado que la población destinataria de este proyecto de formación estaba constituida por el colectivo de personas con discapacidad intelectual de edades comprendidas entre los I 2 y los 20 años, aunque también había actuaciones dirigidas a familiares y a profesionales. Las actividades programadas para las personas con discapacidad intelectual se desarrollaron, en esta fase piloto, en una muestra de 52 jóvenes con síndrome de Down y con otras discapacidades intelectuales que pertenecían a Down Huesca y Down Lleida. La muestra de alumnos que ha participado en el proyecto y el lugar en el que se ubicaban se recoge la Tabla I.

Tabla 1. Alumnos participantes en el proyecto
\begin{tabular}{|l|c|c|}
\hline Provincia & Localidad & $\mathbf{N}^{\mathbf{0}}$ de jóvenes \\
\hline \multirow{2}{*}{ Huesca } & Huesca & 15 \\
\cline { 2 - 3 } & Monzón & 10 \\
\cline { 2 - 3 } & Barbastro & 6 \\
\hline Lérida & Lérida & 21 \\
\hline Total & & $\mathbf{5 2}$ \\
\hline
\end{tabular}

Fuente: Elaboración propia.

\section{Resultados}

\subsection{Con respecto al diseño del programa de formación}

A lo largo del periodo de aplicación del programa de formación, se estableció un primer diseño curricular centrado en la inclusión social y la vida independiente, que contiene los siguientes módulos, en cada uno de los cuales se incluyen algunos talleres y programas. $\mathrm{La}$ estructura curricular, diferenciada por niveles, puede verse en el Cuadro I.

Esta primera propuesta curricular, organizada en módulos, permite mantener una estructura flexible, que facilita la adaptación de los contenidos a las necesidades del grupo y a las necesidades personales de cada alumno. Por ello, los contenidos de cada módulo pueden tener distinto formato metodológico, como talleres, programas o bloques de contenido. En cada módulo, se han planteado diferentes unidades didácticas. Algunos contenidos de esta estructura curricular podrán modificarse en el futuro. La ordenación definitiva de la parrilla curricular estará sujeta, como todo, a un proceso de investigación-acción, en el que la fase de reflexión (a partir de la observación sobre la aplicación y resultados) será fundamental. 
Cuadro 1. Propuesta curricular inicial

\begin{tabular}{|c|c|}
\hline Nivel I (12-16 años) & Nivel II (17-20 años) \\
\hline $\begin{array}{l}\text { Comunicación y la creatividad I: } \\
\text { - Taller de Comprensión Lectora y Creatividad I. } \\
\text { - Taller Conversacional I. } \\
\text { - Taller de Periodistas I. } \\
\text { - Programa de Música/Danza. }\end{array}$ & $\begin{array}{l}\text { Comunicación y la creatividad II: } \\
\text { - Taller de Comprensión Lectora y Creatividad II. } \\
\text { - Taller Conversacional II. } \\
\text { - Taller de Periodistas II. } \\
\text { - Programa de Música/Danza. }\end{array}$ \\
\hline $\begin{array}{l}\text { Autodeterminación e Inclusión Social I: } \\
\text { - Programa de Habilidades Sociales, Autonomía } \\
\text { Personal y Autorregulación. } \\
\text { - Educación Afectivo-sexual. }\end{array}$ & $\begin{array}{l}\text { Autodeterminación e Inclusión Social II: } \\
\text { - Programa de Autodeterminación, Participación } \\
\text { Social y Participación Laboral. } \\
\text { - Educación Afectivo-Sexual. }\end{array}$ \\
\hline $\begin{array}{l}\text { Habilidades para la Vida Independiente I: } \\
\text { - Destrezas en el Contexto Familiar I. } \\
\text { - Destrezas en el Contexto Social I. } \\
\text { - Destrezas en el Contexto Laboral I. }\end{array}$ & $\begin{array}{l}\text { Habilidades para la Vida Independiente II: } \\
\text { - Destrezas en el Contexto Familiar II. } \\
\text { - Destrezas en el Contexto Social II. } \\
\text { - Destrezas en el Contexto Laboral II. }\end{array}$ \\
\hline
\end{tabular}

Fuente: Vived et al. (20I2a).

\subsection{Con respecto al desarrollo de las actividades programadas con los alumnos}

\subsubsection{Aplicación de los diferentes módulos, programas $y$ actividades educativas programadas en los términos previstos}

Esta aplicación de las actividades programadas se ha llevado a cabo en la muestra de alumnos de las diferentes localidades. Los módulos que se han trabajado en el programa formativo han sido los siguientes: Programa de Habilidades Sociales, Autonomía Personal y Autorregulación; Taller de Autodeterminación, Participación Social y Participación Laboral; Destrezas en el Contexto Familiar y Social; Talleres de Comunicación y Creatividad; Educación Afectivo-sexual. Como consecuencia de dicha aplicación, se ha observado una mejora de las habilidades para la vida independiente y las habilidades para la participación social de los alumnos/as participantes, evaluadas a través de registros de observación del funcionamiento de los alumnos en las actividades realizadas y a través de cuestionarios de valoración de las diferentes unidades didácticas.

\subsubsection{Determinación de las orientaciones didácticas que guían la labor del profesorado en cada uno de los talleres y programas}

Las orientaciones didácticas para el profesorado se han planteado con arreglo a las dificultades y necesidades de los alumnos, de las características del taller o programa educativo y del modelo didáctico mediacional, que ha sido el referente de actuación pedagógica (dicho modelo ha sido, a su vez, motivo de indagación, como veremos más adelante).

\subsection{Con respecto a la colaboración y coordinación con la familia}

\subsubsection{Determinación de un plan de formación para las familias}

Los padres han participado en el diseño de un plan de formación para las familias, que tiene los siguientes contenidos:

- La escolarización de los alumnos con discapacidad intelectual. Inclusión escolar: 
políticas, problemas y procesos. Situación actual y perspectivas de futuro.

- Participación de los padres en los marcos asociativos. Participación en la escuela. Participación en la asociación de síndrome de Down. Relación de los padres con los profesionales de la educación: encuentros y desencuentros, problemas y soluciones. La comunicación escuela-familia-asociación.

- Aprendizajes escolares básicos: lectura, escritura, matemáticas, uso del ordenador. Dificultades de aprendizaje más comunes en esta etapa.

- Cómo fomentar la comunicación y el lenguaje.

- Desarrollando la autonomía personal, la competencia social y las habilidades para la vida independiente. Tiempo libre y actividades recreativas.

- Problemas de comportamiento. Pautas para una modificación de conducta.

- Personalidad y desarrollo socioafectivo. Educación emocional. La adolescencia. Educación sexual y afectiva.

- Nuevos conceptos y enfoques sobre la discapacidad intelectual. Implicaciones educativas, sociales y laborales.

- Formación profesional: debilidades del sistema educativo. Transición a la vida adulta.

- Atención médica de las personas con discapacidad intelectual. El programa de salud para personas con síndrome de Down.

\subsubsection{Establecimiento de un sistema de relación con las familias}

Se ha establecido un sistema de relación con las familias que constituye un plan de apoyo y un plan de colaboración, con los siguientes elementos:

- Cursos para familiares.

- Encuentros de padres.

- Orientación familiar.

- Colaboración de las familias.

- Las familias se organizan: canalización de la participación de los padres.

- Participación en los programas educativos (diseño, desarrollo y valoración).

\subsubsection{Elaboración de documentos para padres}

Pensando en los padres, se ha elaborado un documento sobre autodeterminación como principio regulador de la atención a las personas con discapacidad intelectual. Lógicamente, este documento servirá también para otras personas, como profesionales o alumnado en prácticas.

\subsection{Colaboración y coordinación entre los profesionales de las asociaciones y otros agentes educativos}

Para canalizar la colaboración y coordinación entre los profesionales de las asociaciones y otros agentes educativos, se han desarrollado:

- Reuniones trimestrales con las respectivas juntas directivas para explicar el proyecto, su evolución y resultados.

- Reuniones mensuales de coordinación y seguimiento entre las personas responsables del proyecto de las entidades participativas.

- Reuniones semanales (en ocasiones, quincenales) de formación, programación y planificación de acciones, evaluación de procesos y resultados, con los profesionales de cada asociación encargados de aplicar los diferentes módulos del programa.

- Actividades de formación de los voluntarios y alumnado en prácticas de distintas facultades universitarias que han colaborado en el desarrollo de algunos módulos.

Además, se ha elaborado un plan de formación para los profesionales, a partir de debates mantenidos con ellos, cuyos puntos básicos son:

- Marcos conceptuales y normativos:

- Modelo social de la discapacidad.

- Nuevos conceptos sobre discapacidad. Implicaciones educativas.

- Nueva legislación sobre discapacidad.

- Proyectos de vida independiente: oportunidades, apoyos y contenidos:

- Proyectos de vida independiente: nuevas oportunidades de desarrollo personal. 
- Empleo con apoyo.

- Vivienda con apoyo.

- Inclusión social y participación en la comunidad.

- Formación permanente en marcos inclusivos.

- Implicaciones educativas:

- Cambios curriculares en los centros escolares.

- Implicaciones en las prácticas profesionales dentro de las asociaciones. Nuevos retos de las organizaciones sociales.

- Modelo didáctico mediacional.

- Prácticas parentales y autonomía personal.

- Formación para la inclusión social y la vida independiente.

\subsection{Con respecto a la colaboración y coordinación con los profesores de los centros escolares}

El Programa de Formación para la Inclusión Escolar y la Vida Independiente se ha explicado al profesorado de los diferentes centros escolares en los que están escolarizados los alumnos participantes. Este contacto se ha establecido a partir del programa de coordinación y seguimiento escolar, con una frecuencia trimestral.

Además, los materiales didácticos y documentos formativos relacionados con los contenidos del programa formativo se han presentado al profesorado de los diferentes centros.

\subsection{Actuaciones de investigación e innovación}

7.6.1. Indagación sobre nuevos planteamientos didácticos, coherentes con los perfiles de aprendizaje de los alumnos

Un ámbito importante que hemos querido indagar son los procedimientos metodológicos utilizados en el desarrollo de los diferentes módulos formativos. Nos ha preocupado el desarrollo metodológico de las actividades, es decir, hemos querido investigar no tanto el qué hacemos (y cómo repercute sobre los alumnos), sino el cómo lo hacemos, cómo actuamos y cómo repercute en el proceso de enseñanzaaprendizaje. En este sentido, hemos centrado la reflexión en el modelo didáctico mediacional. A partir de los principios didácticos que se han expuesto anteriormente y del análisis del papel del profesor como mediador, hemos concretado una serie de orientaciones didácticas (generales y específicas para cada uno de los talleres y programas que configuran el proyecto de formación) y hemos definido la secuencia didáctica que guía el desarrollo de las unidades didácticas. Esta secuencia didáctica tiene los siguientes componentes:

I. Delimitación de objetivos: en cada unidad didáctica se proponen algunos objetivos vinculados al taller o módulo concreto.

II. Desarrollo metodológico de las actividades: se exponen en este apartado los diferentes componentes que debe abordar el profesor/a para responder a los objetivos planteados. Estas actividades, que se desarrollan en grupos pequeños, son las siguientes:

I. Presentación de los organizadores previos.

2. Evaluación de los conocimientos previos.

3. Realización de las tareas previstas: lectura de textos; representaciones sobre diferentes situaciones sociales, laborales y personales; uso de vídeos u otros materiales audiovisuales vinculados con los objetivos de la unidad didáctica; entre otras. Para cada una de las situaciones de aprendizaje, la secuencia es la siguiente:

- Explicación de las situaciones y de la conducta que se va a realizar.

- Ejemplificación, por parte del profesor/a, de la conducta (con ayuda de un colaborador si es preciso), poniendo énfasis en la distinción de lo fundamental y lo accesorio, en la planificación y en la selección de buenos procedimientos o estrategias. 
- Práctica por parte de los alumnos, con la mediación del profesor, que incidirá en los procesos.

- Valoración y refuerzo de la conducta desarrollada.

4. Diálogo y comentarios sobre las actividades realizadas, con énfasis en el aprendizaje estratégico.

5. Aplicación de los aprendizajes a otras situaciones.

6. Consolidación de la organización cognitiva y resumen de los contenidos abordados.

Además de estas actividades grupales se proponen algunas actividades individuales para la consolidación de los aprendizajes:

7. Actividades con el cuaderno de trabajo personal.

8. Sugerencia de tareas para trabajar con la familia.

III. Evaluación: para evaluar el desarrollo de cada unidad didáctica, se pueden utilizar los registros de sesiones, diseñados para cada unidad didáctica. También podemos utilizar los cuestionarios de autoevaluación que realizan los alumnos, así como los cuestionarios de valoración de las unidades didácticas, que cumplimentan los profesores.

Otro aspecto que también hemos indagado es la valoración de la mediación. La interacción mediacional constituye uno de los elementos fundamentales de la experiencia de aprendizaje mediado; esta interacción puede ser susceptible de evaluación, a pesar de sus dificultades. En efecto, en una situación de aprendizaje mediado, cada elemento puede ser objeto de estudio: el alumno, sus reacciones ante los estímulos, las características del estímulo, el mediador, su estilo, su técnica y su interacción. El mediador, como vemos, también puede ser objeto de evaluación. De hecho, a partir de la evaluación de la mediación se puede lograr una progresiva y positiva modificación del propio educador, de forma que pueda adecuar progresivamente sus prácticas pedagógicas a los criterios de mediación definidos por Feuerstein (I986) y que concretan el perfil del mediador como persona facilitadora de la interacción entre el alumno y el medio. Hemos planteado un cuestionario sobre la mediación, que pretende, precisamente, valorar el estilo de interacción del profesor con los alumnos dentro de un modelo didáctico mediacional. Las dimensiones de este cuestionario están relacionadas con los diferentes criterios de la mediación.

\subsubsection{Diseño y valoración sobre la utilidad de un sistema evaluador del programa formativo}

Este sistema de evaluación, que está basado en los estándares profesionales, nos permite identificar los puntos fuertes y débiles del Programa de Formación para la Inclusión Escolar y la Vida Independiente. Se definieron los estándares de calidad que permiten valorar el desarrollo de los distintos programas y talleres. Para cada estándar, se identificaron varios indicadores, que son enunciados en los que se concretan los estándares. Con los indicadores, podemos comparar y contrastar nuestra práctica profesional, valorando en qué medida el programa se adecua a los estándares apuntados. Los estándares nos permiten, por tanto, valorar los programas, pero pueden y deben utilizarse como mecanismos o instrumentos de mejora y homologación de las acciones de los centros. En este sentido, constituyen una ayuda que permite prevenir errores en el diseño y desarrollo de programas, puesto que se elaboran a partir de la identificación de acciones que son consideradas por los profesionales como aceptables y recogen las mejores acciones actuales.

La evaluación de las acciones se realiza través del cuestionario de valoración de los estándares de calidad. Es evidente que, con este planteamiento, se pretende definir mecanismos de actuación que permitan mejorar aquellos indicadores que, a través de la valoración, se hayan considerado deficitarios. No hay que olvidar que el objeto básico de estos estándares es el de contribuir a la reflexión sobre la eficacia de cada componente del programa y poder 
realizar las correcciones precisas que permitan mejorar la calidad de la intervención educativa.

\subsection{Con respecto a la elaboración de material didáctico}

Se han elaborado diversos materiales didácticos vinculados a los diferentes módulos del programa formativo. Los hemos organizado en dos categorías: los que se han publicado, y los que hemos maquetado y se incorporan a la página web de Down España para que se puedan descargar, en función de las necesidades.

- Publicaciones:

- Taller de Comprensión Lectora: habilidades sociales, autonomía personal y autorregulación. Textos para el alumno.

- Taller de Comprensión Lectora: habilidades sociales, autonomía personal y autorregulación. Cuaderno personal para el alumno.

- Taller de Comprensión Lectora: autodeterminación, participación social y laboral. Textos para el alumno.

- Edición y maquetación:

- Taller de Comprensión Lectora: habilidades sociales, autonomía personal y autorregulación. Orientaciones didácticas.

- Taller de Comprensión Lectora: habilidades sociales, autonomía personal y autorregulación. Material de evaluación y seguimiento.

- Taller de Comprensión Lectora: autodeterminación, participación social $y$ participación laboral. Orientaciones didácticas.

- Taller de Comprensión Lectora: autodeterminación, participación social y participación laboral. Material de evaluación y seguimiento.

- Taller de Comprensión Lectora y Creatividad Literaria (I): mi primer libro de relatos, poesía y teatro. Textos para el alumno.
- Taller de Comprensión Lectora y Creatividad Literaria (I): mi primer libro de relatos, poesía y teatro. Orientaciones didácticas.

- Taller de Periodistas (I): mi primer libro de noticias y entrevistas. Textos para el alumno.

- Taller de Periodistas (I): mi primer libro de noticias y entrevistas. Orientaciones didácticas.

\subsection{Con respecto a las actuaciones de} divulgación

- Comunicaciones en congresos: VIII Jornadas Científicas de Investigación sobre Personas con Discapacidad, organizadas en Salamanca los días I4, I 5 y I 6 de marzo de 2012 por el Instituto de Integración en la Comunidad (Universidad de Salamanca); XII Encuentro de Familias de Personas con Síndrome de Down, organizadas por Down España en Huesca los días I-4 de noviembre de 2012.

- Publicación de artículo en revista especializada: Vived et al. (2012a).

- Participación en cursos de formación: 'Formación para el empleo y discapacidad', impartido dentro de los cursos de verano de la Universidad de Zaragoza, en Jaca, los días 9 y ro de julio de 20 I 2.

- Diseño de los contenidos de formación para profesionales de la Red Nacional de Educación de Down España.

\section{Algunas conclusiones}

I. Este proyecto tiene un marcado carácter innovador, que trata de poner en práctica la nueva conceptualización sobre discapacidad y los nuevos planteamientos sobre los proyectos de vida de las personas con síndrome de Down o con otras discapacidades intelectuales. La innovación también se extiende al enfoque pedagógico, 
centrado en el modelo didáctico mediacional; al diseño de materiales didácticos, en cuya elaboración se ha utilizado la metodología de lectura fácil; y al sistema de valoración del programa, que se basa en los estándares de calidad.

2. El desarrollo de los proyectos de vida independiente va a depender, en gran medida, de la definición de oportunidades adecuadas y apoyos que respondan a las necesidades de las personas con discapacidad intelectual. En este sentido, ya hemos comentado que estas oportunidades se pueden concretar en, al menos, los siguientes entornos inclusivos:

- Un entorno de vivienda que podemos definir como piso de vida independiente (o vivienda con apoyo).

- Un entorno laboral normalizado, donde los jóvenes con discapacidad intelectual puedan acceder al empleo a través de metodologías adecuadas (empleo con apoyo).

- Entornos comunitarios accesibles que permitan la participación de todos los ciudadanos, también de los ciudadanos con discapacidad intelectual.

- Entornos formativos inclusivos, que ofrezcan currículos apropiados a las características y necesidades personales, socioculturales y laborales de los jóvenes con discapacidad intelectual.

3. Los nuevos conceptos y principios mencionados deben orientar nuestras acciones, y modificar el funcionamiento de los servicios y sistemas de atención a las personas con discapacidad. A los profesionales, a los familiares, y a las instituciones públicas y privadas que apoyan a las personas con discapacidad nos corresponde el reto de transformar las prácticas profesionales y parentales, los programas y servicios en función de los nuevos conceptos, para poder ofrecer una mayor calidad de vida a las personas con discapacidad intelectual.
4. Los proyectos de vida independiente deben ser referentes de las propuestas formativas que se dirigen a las personas en las etapas previas a su emancipación familiar (con los apoyos pertinentes en cada caso), es decir, en las últimas etapas de su itinerario educativo. Dicho de otra manera, es necesario establecer, dentro de la respuesta educativa que reciben los alumnos, una línea formativa orientada a la vida independiente. Y ésta es la razón del proyecto que presentamos.

5. Además del impulso en el desarrollo de estos ámbitos contextuales (vivienda, trabajo, comunidad, formación), que van a permitir el desarrollo de itinerarios individuales de vida independiente, basados en la autodeterminación y en la inclusión social, se requiere también una formación específica para la inclusión social y la vida independiente que se presente con los nuevos planteamientos teóricos que hemos definido y con una metodología didáctica mediacional, basada en el sentimiento de competencia, en los procesos y en la generalización de los aprendizajes. Dicha formación debe ir dirigida tanto a las personas con síndrome de Down o con otra discapacidad intelectual, como a sus familias y a los profesores y técnicos que se relacionan con ellas.

6. El Programa para la Inclusión Social y la Vida Independiente se está mostrando como un programa orientado al desarrollo de varias habilidades personales necesarias para fomentar la autonomía personal y una disposición favorable a participar en la sociedad. La aplicación del programa incide en el desarrollo de un itinerario individualizado hacia la inclusión social y la vida independiente.

7. En lo que hace referencia a la colaboración con la familia, la participación de los padres constituye un elemento esencial para el éxito de la intervención educativa en los niños y jóvenes con discapacidad intelectual. Que los padres se integren 
en el proceso de la educación de sus hijos permitirá optimizar la intervención educativa. Son enormes las ventajas de la participación activa de la familia en los programas educativos de los jóvenes con discapacidad intelectual. Cuando los padres están implicados en los programas de intervención, el mantenimiento y generalización de los aprendizajes hechos por el niño o el joven tienen más posibilidades de producirse y consolidarse. En el presente programa, se plantea la colaboración de los padres en tres ámbitos importantes: en la aplicación del programa, en la valoración del desarrollo del hijo y en la valoración del programa.

8. Por lo que respecta a los centros escolares, una de las finalidades de este proyecto fue el desarrollo de mecanismos de colaboración entre las asociaciones de síndrome de Down con los centros educativos para ofrecer una respuesta educativa integral y coordinada, centrada en la satisfacción de las necesidades educativas de los alumnos con discapacidad intelectual y coherente con los nuevos conocimientos y conceptos sobre discapacidad en general y discapacidad intelectual en particular.

9. Impulso de redes internas y externas. Es éste un proyecto que ha planteado el aprendizaje cooperativo como una estructura importante para la enseñanza de los contenidos del programa, pero también queremos reivindicar el aprendizaje cooperativo como una filosofía de funcionamiento y de relación interpersonal. La interdependencia positiva y solidaria debe ser una consideración clave en el desarrollo del proyecto, de tal manera que una línea nueva de reflexión debe estar centrada en nuevos modos de definir la cooperación entre distintos agentes educativos. Nuevos conceptos, nuevas expectativas, nuevos planteamientos educativos, nuevos proyectos de vida para las personas con discapacidad intelectual requieren de nuevas competencias profesionales, nuevas prácticas parentales y nuevos modos de definir la cooperación entre los distintos agentes. La cultura de funcionamiento en red debe también mejorarse. Los marcos de colaboración que se definen en este proyecto son los siguientes:

- Entre profesionales de distintas entidades de la Red Nacional de Educación Down.

- Entre profesionales y familias.

- Entre asociaciones y centros escolares (incluyendo en esta vinculación los centros de profesores y recursos, $y$ otros servicios educativos).

- Entre asociaciones y universidades.

- Con los medios de comunicación.

- Con y entre distintas administraciones (locales, autonómicas y nacionales; educativas, sociales y culturales).

Io. Propuestas de mejora. A través del cuestionario de valoración del programa, basado en los estándares profesionales se pretende definir mecanismos de actuación que permitan mejorar aquellos indicadores que, a través de la valoración, se hayan considerado deficitarios. No hay que olvidar que el objeto básico de estos estándares es el de contribuir a la reflexión sobre la eficacia de cada componente del programa y poder realizar las correcciones precisas que permitan mejorar la calidad de la intervención educativa. 
Alonso, F. (2007): "La accesibilidad universal y el diseño para todos", en De Lorenzo, R. y Cayo, L. (coords.), Tratado sobre discapacidad, Cizur Menor: Aranzadi.

Feuerstein, R. (I996): "La teoría de la modificabilidad estructural cognitiva", en Molina, S. y Fandos, M. (coords.), Educación cognitiva, vol. I, Zaragoza: Mira Editores.

- (I986): Mediated Learning Experience, Yerushaláyim: Hadassah-Wizo Canada Research Institute.

Foro de Vida Independiente (en línea), <http:// www.forovidaindependiente.org>.

Ginnerup, S. (2010): Hacia la plena participación mediante el diseño universal, Madrid: Imserso.

Haywood, H.C. et al. (1992): Bright Start: Cognitive Curriculum for Young Children, Watertown: Charlesbridge Publishers (edición española: Bright Start, curriculum cognitivo para niños, Zaragoza: FUNDAFE, I997).

Jiménez, A. (2007): “Conceptos y tipologías de la discapacidad”, en De Lorenzo, R. y Cayo, L. (coord.), Tratado sobre discapacidad, Cizur Menor: Aranzadi.

Luckasson, R., et al. (2002): Mental Retardation. Definition, Classification and Systems of Supports, Washington, D.C.: American Association on Mental Retardation (edición española: Verdugo, M.A. y Jenaro, C., Retraso mental. Definición, clasificación y sistemas de apoyo, Madrid. Alianza Editorial, 2004).

Molina, S. et al. (2008a): Programa para el desarrollo de habilidades básicas en alumnos con sindrome de Down, Zaragoza: PUZ.

Molina, S. et al. (2008b): "Experimentación de la eficacia de un modelo didáctico en una muestra de niños con síndrome de Down", Revista de Investigación Educación y Diversidad, 2: 63IOI

Prieto, M.D. (1989): Modificabilidad cognitiva y P.E.I., Madrid: Bruño.
Schalock, R.L. (2009): "La nueva definición de discapacidad intelectual, los apoyos individuales y los resultados personales”, en Verdugo, M. A. et al. (coords.), Mejorando resultados personales para una vida de calidad, Salamanca: Amarú.

- (I996): Quality of Life: Vol I. Conceptualitation and Measurement, Washington, D.C.: American Association on Mental Retardation.

Schalock, R.L. et al. (2010): Intellectual Disability. Definition, Classification and Systems of Supports ( I $^{\text {th }}$ Edition), Washington, D.C.: American Association on Intellectual and Developmental Disabilities.

Van Loon, J.H.M. (2008): Aligning Quality of Life Domains and Indicators to Support Intensity Scale Companion Guide: A Resource for SIS User, Washington, D.C.: American Association on Intellectual and Developmental Disabilities.

Verdugo, M.A. (20IO): "Últimos avances en el enfoque y concepción de las personas con discapacidad intelectual", Siglo Cero, 4I (4): 7-2I.

- (I995): "Personas con retraso mental", en Verdugo, M.A. (dir.), Personas con discapacidad. Perspectivas psicopedagógicas y rehabilitadoras, Madrid: Siglo XXI.

Vidal, J. (2003): El movimiento de vida independiente, Madrid: Fundación Luis Vives.

Vigotsky, L.S. (1979): El desarrollo de los procesos psicológicos superiores, Barcelona: Crítica; Grijalbo.

- (1964): Pensamiento y lenguaje, Buenos Aires: Lautaro,

Vived, E. (20I I): Habilidades sociales, autonomía personal y autorregulación, Zaragoza: Prensas Universitarias de Zaragoza.

Vived, E. et al. (201 2a): "Formación para la inclusión social y la vida independiente", Revista Síndrome Down Adultos, I I: 2-I4.

Vived, E. et al. (201 2b): Autodeterminación, participación social y participación laboral, Zaragoza: Mira Editores. 
Wehmeyer, M.L. (1996): “Self-determination in youth with severe cognitive disabilities: From theory to practice", en Powers, L., et al. (eds.), Making Your Way: Building Self-competence among Children and Youth with Disabilities, Baltimore: Brookes.
Wehmeyer, M.L., et al. (2006): Escala de Autodeterminación Personal ARC. Instrumento de valoración y guía de aplicación, Madrid: CEPE. 